\title{
3-Nitrotyrosine, a biomarker for cardiomyocyte apoptosis induced by diabetic cardiomyopathy in a rat model
}

\author{
YA-LI ZHANG ${ }^{1}$ and JIN-RU WEI ${ }^{2}$ \\ ${ }^{1}$ Department of Cardiovascular Disease, The Fifth Affiliated Hospital of Guangxi Medical University, \\ Liuzhou, Guangxi 545006; ${ }^{2}$ Department of Cardiovascular Disease, The First People's Hospital of Nanning City, \\ Nanning, Guangxi 530022, P.R. China
}

Received December 21, 2012; Accepted April 16, 2013

DOI: $10.3892 / \mathrm{mmr} .2013 .1644$

\begin{abstract}
The aim of this study was to investigate whether 3-nitrotyrosine (3-NT) may serve as a predictive biomarker for cardiomyocyte apoptosis in a diabetic cardiomyopathic rat model. Using male Sprague-Dawley (SD) rats in a prospective, randomized, controlled study, a diabetic type II animal model was established by injection with streptozotocin. The diabetic group and the diabetic cardiomyopathy (DCM) group were administered valsartan. This study revealed the following: i) The ratio of heart/body weight increased in the DCM, diabetes administered with valsartan $(\mathrm{D}+\mathrm{V})$ and $\mathrm{DCM}+\mathrm{V}$ groups compared with the $\mathrm{N}$ group. ii) The expression index (EI) of 3-NT correlated positively with the apoptotic index (AI) of the cardiomyocytes, whereas 3-NT in the serum did not reflect changes in the AI. iii) The AI of the DCM group was the highest of the assessed groups. The AI of the DCM group was higher than that in the $\mathrm{D}+\mathrm{V}$ group, and the $\mathrm{AI}$ of the $\mathrm{DCM}+\mathrm{V}$ group was higher than that of the $\mathrm{N}$ group. iv) The EI of 3-NT increased with a higher AI. The higher the EI of 3-NT, the higher the AI observed in rat cardiomyocytes. Both the EI of 3-NT and the AI of the DCM group were higher than in the other groups. However, both the EI of 3-NT and the AI in the DCM group markedly decreased for the DCM+V group. 3-NT in the myocardial tissue of rats was deemed to be a successful biomarker for predicting cardiomyocyte apoptosis in SD rats. 3-NT levels were significantly increased and related to cardiomyocyte apoptosis. Valsartan post-treatment reversed the increase of 3-NT and apoptosis in diabetic cardiomyopathy.
\end{abstract}

\section{Introduction}

Diabetic cardiomyopathy (DCM) is a unique, chronic and progressive heart disease with cardiac muscle dysfunc-

Correspondence to: Dr Jin-Ru Wei, Department of Cardiovascular Disease, The First People's Hospital of Nanning City, 89 QiXing Road, Nanning, Guangxi 530022, P.R. China

E-mail: jinruweidj@yahoo.cn

Key words: 3-nitrotyrosine, diabetic cardiomyopathy, apoptosis tion and a long-term latent phase induced by diabetes. The pathological alterations of DCM vary from other heart diseases, such as hypertension and coronary artery disease, although the majority of patients with DCM encounter earlier signs, including hypertension and ischemic chest pain. DCM is characterized by ventricular dilation, myocyte hypertrophy and interstitial fibrosis, and combines with decreased systolic function and diastolic dysfunction (1).

It is known that the complications in diabetes derive from hyperglycemia. Several factors have been demonstrated to be involved in the pathogenesis of DCM. Etiologically, there are four primary factors that contribute towards heart failure in DCM: i) Microangiopathy and related endothelial dysfunction; ii) autonomic neuropathy; iii) metabolic alterations (abnormal hyperglycemia and fatty acid hyperoxidation); and iv) the accumulation of free radicals (reactive oxygen/nitrogen species, ROS/RNS) (2).

Previously, the mechanism for DCM was established, and was shown to be an acute reaction of cardiac myocytes, induced by hyperglycemia, that gradually develops into chronic pathological alterations, including abnormal gene expression, disorder of signaling pathways and aggravated cellular apoptosis (3). Hyperglycemia, derived from metabolic alteration, may lead to a cascade of endovascular reactions, including the release of inflammatory cytokines, chemo-attractant infiltration of monocytes and elevated angiotensin II levels. The activation of angiotensin II receptors 1 and 2 (AT1 and AT2) by angiotensin II provokes oxidative stress, induces cardiac inflammation and produces a highly toxic peroxynitrite $\left(\mathrm{RNS}, \mathrm{ONOO}^{-}\right)$(4). Free tyrosine and proteins with a tyrosine amino acid are rapidly nitrated by $\mathrm{ONOO}^{-}$, resulting in the formation of the end-product 3-nitrotyrosine (3-NT), which impairs cardiac mitochondrial function (5).

The primary aim of this study was to investigate whether 3-NT is a potential biomarker for DCM in cardiac tissue and serum, and to establish the correlation between 3-NT and the apoptosis of cardiomyocytes.

\section{Materials and methods}

Materials. Streptozotocin (STZ) was purchased from Sigma (St.Louis, MO, USA). The citrate buffer vehicle ( $\mathrm{pH} 4$.2-4.5) was prepared by mixing solution A ( $2.1 \%$ citric acid) and solution $\mathrm{B}$ 
(2.94\% sodium citrate) at a ratio of 1:1.32. An injective solution of STZ was prepared by dissolving $1 \mathrm{~g}$ in $100 \mathrm{ml}$ of the citrate buffer. Anti-3-NT rat polyclonal antibodies were purchased from Abcam (Cambridge, MA, USA) (6). The TUNEL reagent kit was purchased from Roche (South San Francisco, CA, USA). The rat 3-NT ELISA kit was purchased from Wuhan USCN Sciences Co., Ltd. (Wuhan, China).

The color development reagent was purchased from Gui Hai Bio-Tech Inc. (Nanning, China). Rat blood glucose levels were measured using a VAC0037941 blood glucose meter (Taiwan Leader Bio-Tech Inc., Taipei City, Taiwan, R.O.C.). Valsartan capsules were provided by Tianda Pharmaceutical Inc. (Zhuhai, China).

\section{Methods}

Study design. A total of 70 male Sprague-Dawley (SD) rats (8 weeks old; body weight, 180-220 g) were purchased from the Animal Breed Center affiliated with Guangxi Medical University (Nanning, China). The rats were housed individually in polycarbonate cages and the animal room was conditioned according to National Standard Regulations and The Association For Assessment and Accreditation of Laboratory Animal Care International (AAALAC) requirements. The room temperature was set to $23 \pm 3^{\circ} \mathrm{C}$, the relative humidity at $50 \pm 2$, the air ventilation at $10-15$ changes $/ \mathrm{h}$ and a light cycle of $12 \mathrm{~h}$ on, $12 \mathrm{~h}$ off. The rats were randomly allocated into four groups based on recent body weight (n=15/group).

The $N$ group. In this control group, $20 \mathrm{SD}$ rats were given free access to a standard pellet diet and distilled water. The rats were injected intraperitoneally (i.p.) with citrate buffer solution. For screening, 5 rats were used as a comparison with the DCM rats, and 15 were assigned to the end point of the study.

A further $60 \mathrm{SD}$ rats were used to establish the type II diabetic animal model. These rats were fed a hyperlipid and hyperglycemia diet (consisting of $10 \%$ pork oil, $10 \%$ sucrose and 5\% egg yolk) for 4 weeks. Afterwards, the rats fasted overnight and each rat was i.p. injected once with STZ at $40 \mathrm{mg} / \mathrm{kg}$ body weight to establish a type II diabetic rat model. These rats were diagnosed as type II diabetic rats, as determined by a higher blood glucose level ( $\geq 16.7 \mathrm{mmol} / \mathrm{l})$ measured via the tail vein.

The $D+V$ group. In this group, 15 type II diabetic SD rats were gavaged with a valsartan capsule at $40 \mathrm{mg} / \mathrm{kg}$ body weight every day for 4 weeks, retaining free access to a standard rodent diet and water.

A further 45 type II diabetic rats were fed continuously on a hyper-lipid and hyperglycemia diet for a further 4 weeks. Afterwards, 5 rats were randomly selected and sacrificed for comparison with 5 control rats. The absolute heart weight and the ratio of heart/body weight were found to be higher than in the control rats. Immunohistological staining of the heart slices revealed swelling and necrosis of the cardiomyocytes, proliferation of the interstitial fibroblast cells, over-growth of the collagen fibers and disassembly of cellular organization. These rats were diagnosed as DCM rats and used in the further study.

The DCM group. In this group, 15 DCM rats were selected from the above process, assigned into the DCM group for the study and were fed the hyper-lipid and hyperglycemia diet for another 4 weeks.

The DCM+V group. In this group, 15 DCM rats were fed a standard diet, but were gavaged with a valsartan capsule at $40 \mathrm{mg} / \mathrm{kg}$ body weight every day for a further 4 weeks.

After the 4 weeks, the rats in all four experimental groups were sacrificed by $\mathrm{CO}_{2}$ inhalation to end the in vivo study.

Measurement of animal heart weight. Once the rats were euthanized, $3 \mathrm{ml}$ of blood was drawn from the aorta of each rat. Serum was isolated by centrifugation for $10 \mathrm{~min}$ at $1500 \mathrm{x} \mathrm{g}$ at $4{ }^{\circ} \mathrm{C}$, and frozen immediately at $-70{ }^{\circ} \mathrm{C}$ for the ELISA assay at a later time. The rat heart was quickly removed and weighed after the blood had been absorbed by filter paper. The ratio of heart/body weight was calculated.

Histological staining and TUNEL assay. After weighing the hearts, they were perfused with $4 \%$ paraformaldehyde for fixation. After fixation, the samples underwent a series of gradient dehydrations, and were embedded in paraffin and sliced for hemotoxylin and eosin (H\&E) staining.

The TUNEL assay was performed according to the manufacturer's instructions. Under a phase contrast microscope (CHC-212 digital microscope; Olympus Optical, Co. Ltd., Tokyo, Japan), cells with a yellow-brown nucleus were deemed to be positie for apoptosis. Using x400 magnification, 5 representative fields were randomly selected. The apoptotic index (AI) was calculated from the number of apoptotic cells per 100 cells. The amount of 3-NT in the myocardial tissue was determined by immunostaining, and high definition images were captured using an Olympus CHC-212 digital microscope and statistically analyzed with Motic Images Advanced 3.2 software (Motic China Group Co. Ltd., Xiamen, China). The mean optical density (MOD) of positively stained apoptotic nuclei was calculated and the percentage positive expression of nucleus area was acquired by dividing the positively stained area by the entire nucleus area. The expression index (EI) of 3-NT in myocardial tissue was calculated as follows: MOD x positive area (\%) x 100 (7).

Blood concentration of 3-NT. The blood concentration of 3-NT was detected using the ELISA method. The OD value of 3-NT via color development was determined at $450 \mathrm{~nm}$ using a spectrometer. The concentration of $3-\mathrm{NT}$ in the blood was determined using a standard curve.

Statistical analysis. All the data are expressed as the mean \pm SD and underwent statistical analysis with SPSS 13.0 software (IBM SPSS, Armonk, NY, USA). P<0.05 was considered to indicate a statistically significant difference in comparison with the control.

\section{Results}

Moribund and dead animals during the study. A total of 7 animals died while the type II diabetic model was being established and prior to entry to the DCM group; three rats died while being medicated in the $\mathrm{D}+\mathrm{V}$ group, two died while being prepared for DCM, one rat died while being prepared 
Table I. Results of heart/weight index.

\begin{tabular}{lll}
\hline Group & $\mathrm{n}$ & Heart/body weight ratio \\
\hline $\mathrm{N}$ & 15 & $0.002824 \pm 0.000304$ \\
$\mathrm{DCM}$ & 13 & $0.004504 \pm 0.001184^{\mathrm{a}}$ \\
$\mathrm{D}+\mathrm{V}$ & 12 & $0.003271 \pm 0.000364$ \\
$\mathrm{DCM}+\mathrm{V}$ & 10 & $0.005181 \pm 0.001330^{\mathrm{a}}$
\end{tabular}

${ }^{\mathrm{a}} \mathrm{P}<0.05$ vs. the normal group. Results are expressed as the mean $\pm \mathrm{SD}$. N, normal group; DCM, diabetic cardiomyopathy group; $\mathrm{D}+\mathrm{V}$, diabetes + valsartan pretreatment group; $\mathrm{DCM}+\mathrm{V}$, diabetic cardiomyopathy + valsartan treatment group.

Table II. Comparison of apoptotic index (AI) in each group.

\begin{tabular}{lcc}
\hline Group & $\mathrm{n}$ & $\mathrm{AI}$ \\
\hline $\mathrm{N}$ & 15 & $0.272 \pm 0.155$ \\
$\mathrm{DCM}$ & 13 & $63.705 \pm 26.341^{\mathrm{a}}$ \\
$\mathrm{D}+\mathrm{V}$ & 12 & $23.876 \pm 18.385^{\mathrm{b}}$ \\
$\mathrm{DCM}+\mathrm{V}$ & 10 & $14.077 \pm 11.140^{\mathrm{b}}$ \\
\hline
\end{tabular}

${ }^{\mathrm{a}} \mathrm{P}<0.01$ vs. the normal group $(\mathrm{N})$; ${ }^{\mathrm{b}} \mathrm{P}<0.05$ vs. the normal group. Results are expressed as the mean \pm SD. DCM, diabetic cardiomyopathy group; $\mathrm{D}+\mathrm{V}$, diabetes + valsartan pretreatment group; $\mathrm{DCM}+\mathrm{V}$, diabetic cardiomyopathy + valsartan treatment group.

Table III. Correlation analysis of the expression index (EI) of $3-\mathrm{NT}$ in the heart and the apoptotic index (AI).

\begin{tabular}{lccc}
\hline Group & $\mathrm{n}$ & EI & AI (r) \\
\hline $\mathrm{N}$ & 15 & $46.749 \pm 30.295$ & $0.272 \pm 0.155\left(0.805^{\mathrm{a}}\right)$ \\
$\mathrm{DCM}$ & 13 & $966.204 \pm 555.885$ & $63.705 \pm 26.341\left(0.807^{\mathrm{a}}\right)$ \\
$\mathrm{D}+\mathrm{V}$ & 12 & $370.173 \pm 297.776$ & $23.876 \pm 18.385\left(0.972^{\mathrm{a}}\right)$ \\
DCM+V & 10 & $342.508 \pm 227.516$ & $14.077 \pm 11.140\left(0.965^{\mathrm{a}}\right)$
\end{tabular}

${ }^{\mathrm{a}} \mathrm{P}<0.01$ for comparisons between all groups. Results are expressed as the mean $\pm \mathrm{SD}$. 3-NT, 3-nitrotyrosine; r, correlation coefficient; $\mathrm{N}$, normal group; DCM, diabetic cardiomyopathy group; $\mathrm{D}+\mathrm{V}$, diabetes + valsartan pretreatment group; $\mathrm{DCM}+\mathrm{V}$, diabetic cardiomyopathy + valsartan treatment group.

for the diabetes group and one rat died due to an accident during valsartan capsule gavage. A further 10 rats were sacrificed to measuere their heart weights. Of the $\mathrm{DCM}+\mathrm{V}$ group, 3 rats were observed not to have been induced into DCM, according to hematoxylin and eosin-stained heart sections and the TUNEL assay results of myocardial apoptosis. A total of 50 animals survived the study, leaving 15 rats in the $\mathrm{N}$ group, 13 rats in the DCM group, 12 rats in the $\mathrm{D}+\mathrm{V}$ group and 10 rats in the $\mathrm{DCM}+\mathrm{V}$ group.

General physical observation. Diabetic rats were found to be thin or emaciated and weak, and they walked at a slow and clumsy pace. They drank an increased amount of water, resulting in an increase in urine (approximately twice more than rats in the $\mathrm{N}$ group). Diabetic rats had filthy depleted fur. Blood glucose was maintained at $>16.7 \mathrm{mmol} / \mathrm{l}$ in diabetic rats, whereas in the control rats the blood glucose levels were found to be $<6.0 \mathrm{mmol} / 1$.

Ratios of heart/body weight in the four groups were analyzed statistically with ANOVA, and statistically significant differences were found between the groups $(\mathrm{F}=18.63$, $\mathrm{P}<0.01)$. The ratios for the DCM group were higher than those in the $\mathrm{N}$ group, and the ratios for the $\mathrm{DCM}+\mathrm{V}$ group were higher than those in the $\mathrm{D}+\mathrm{V}$ group $(\mathrm{P}<0.01$; Table I.)

Effects of valsartan on cardiomyocyte structure examined using histopathology. As shown in Fig. 1, the cardiomyocytes were assembled neatly, and the sizes of the cell bodies and nuclei were similar. H\&E-stained plasma revealed that the intracellular staining was even and smooth, and the plasma membrane was intact. However, varied degradation and necrosis of cardiomyocytes, proliferation of intercellular fibroblasts, increased collagen levels and cell disarrangement were observed in the DCM group.

Effects of valsartan on cardiomyocyte apoptosis examined using histopathology. Cardiomyocyte apoptosis was examined using the TUNEL method. As shown in Fig. 2, for the DCM group, cellular nuclei stained brown or dark yellow were considered to be positive for apoptosis when examined under a phase contrast microscope. AI was deemed to be statistically significant between the four groups $(\mathrm{F}=34.779$; $\mathrm{P}<0.01$; Table II).

Distribution of the EI for 3-NT among different cardiomyocytes examined by histopathology. As shown in Fig. 3, plasma area stained a yellow-brown color indicates a higher EI for 3-NT, which is a strong reaction. There was a high EI for 3-NT in the DCM group, and a weak reaction or lower EI for 3-NT in the $\mathrm{D}+\mathrm{V}$ and $\mathrm{DCM}+\mathrm{V}$ groups. However, there was a negative EI for 3-NT in the N group (background color was light blue). As shown in Table III, the EIs for 3-NT correlated positively with AI among all the tested groups. As shown in Table IV, the EIs for 3-NT in the DCM, D+V and DCM+V groups were markedly higher than in the $\mathrm{N}$ group. The EI value for the $\mathrm{DCM}+\mathrm{V}$ group was more than half that of the DCM group.

Correlation of blood 3-NT with the AI of cardiomyocytes. In Table V, the correlation factor $r$ between blood 3-NT and AI was very small or negative. In contrast to Table III, it appears that blood 3-NT was unrelated to the AI in cardiomyocytes.

\section{Discussion}

In this study, 3-NT in the myocardial tissue was deemed to be a successful biomarker for predicting cardiomyocyte apoptosis in SD rats. 3-NT levels were increased and were closely related to cardiomyocyte apoptosis. Post-treatment with valsartan reversed the increase in 3-NT levels and apoptosis in diabetic cardiomyopathic cells.

It was demonstrated that the hearts of rats in the DCM group were larger than those in the $\mathrm{N}$ group. After receiving 

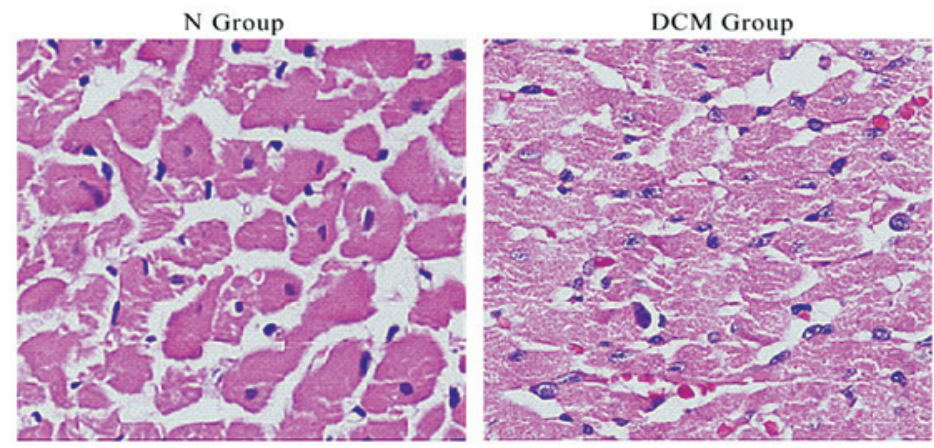

Figure 1. Hematoxylin and eosin-stained heart sections from rats of the normal (N) and diabetic cardiomyopathy (DCM) groups (magnification, $\mathrm{x} 400)$.
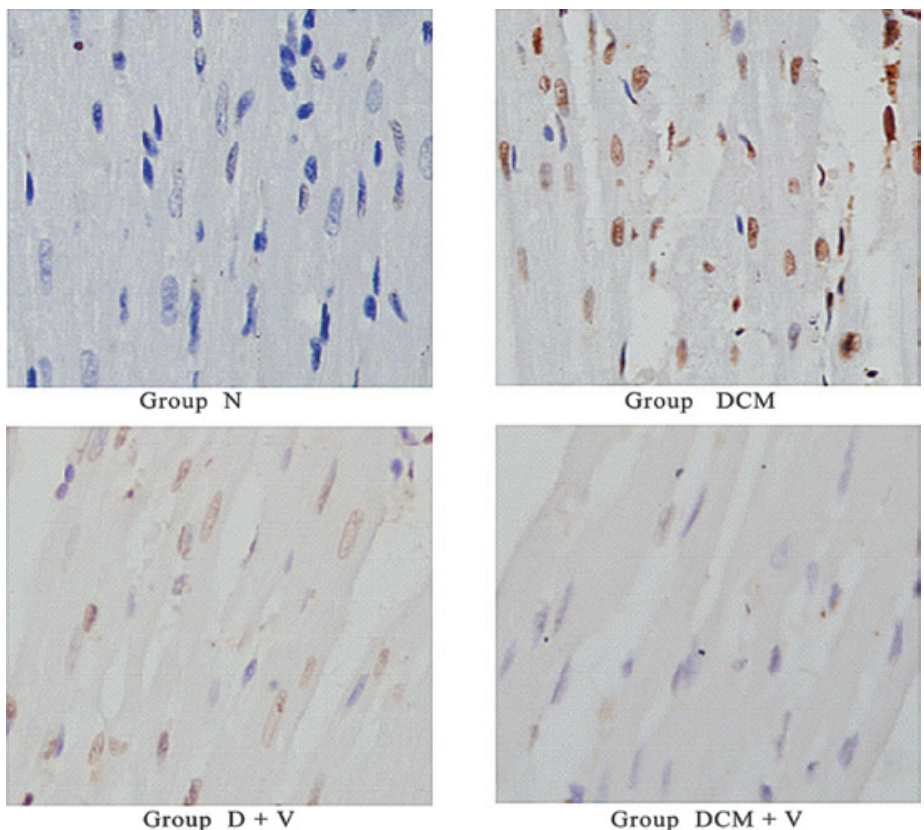

Figure 2. TUNEL assay results of myocardial apoptosis (magnification, $\mathrm{x} 400$ ). N, normal group; DCM, diabetic cardiomyopathy group; D+V, diabetes + valsartan pretreatment group; DCM+V, diabetic cardiomyopathy + valsartan treatment group.
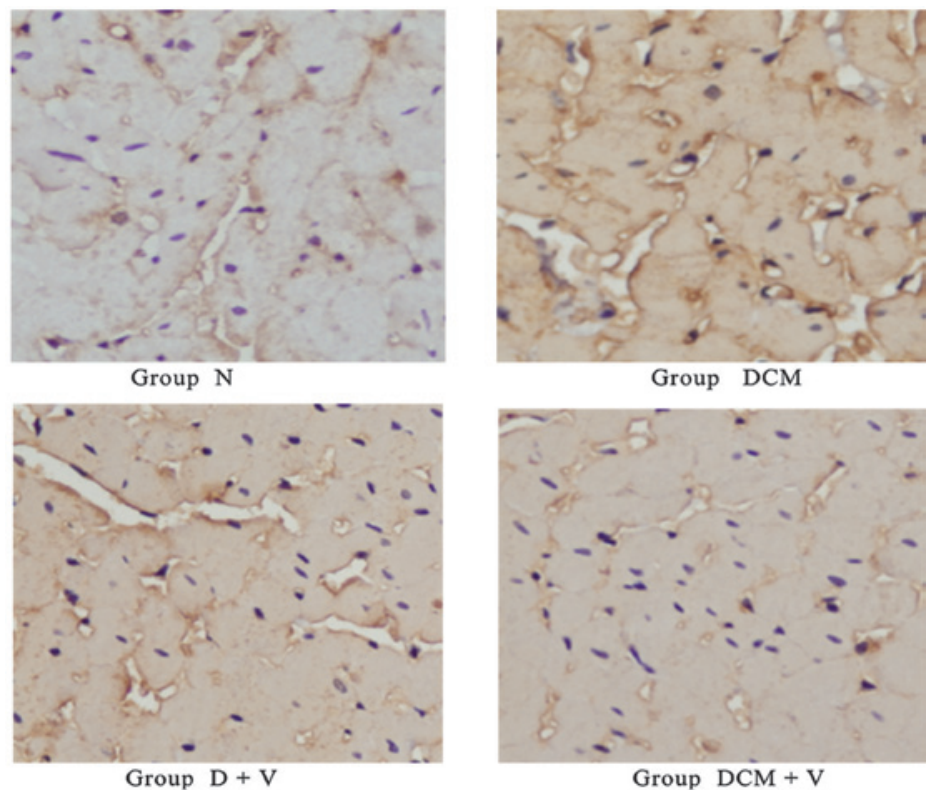

Figure 3. Immunohistochemical detection of 3-NT in myocardial cells (magnification, x400). 3-NT, 3-nitrotyrosine; N, normal group; DCM, diabetic cardiomyopathy group; $\mathrm{D}+\mathrm{V}$, diabetes + valsartan pretreatment group; $\mathrm{DCM}+\mathrm{V}$, diabetic cardiomyopathy + valsartan treatment group. 
Table IV. Comparison of the EI of 3-NT in the heart.

\begin{tabular}{lcc}
\hline Group & $\mathrm{n}$ & $\mathrm{EI}$ \\
\hline $\mathrm{N}$ & 15 & $46.749 \pm 30.295$ \\
$\mathrm{DCM}$ & 13 & $966.204 \pm 555.885^{\mathrm{a}}$ \\
$\mathrm{D}+\mathrm{V}$ & 12 & $370.173 \pm 297.776$ \\
$\mathrm{DCM}+\mathrm{V}$ & 10 & $342.508 \pm 227.516$
\end{tabular}

${ }^{\text {ap }}<0.05$ vs. the normal group. Results are expressed as the mean $\pm \mathrm{SD}$ $\mathrm{EI}$, expression index; 3-NT, 3-nitrotyrosine; N, normal group; $\mathrm{DCM}$, diabetic cardiomyopathy group; $\mathrm{D}+\mathrm{V}$, diabetes + valsartan pretreatment group; $\mathrm{DCM}+\mathrm{V}$, diabetic cardiomyopathy + valsartan treatment group.

Table V. 3-NT concentration and correlation analysis of AI.

\begin{tabular}{lccc}
\hline Group & $\mathrm{n}$ & 3-NT & AI (r) conc. (nmol/l) \\
\hline $\mathrm{N}$ & 10 & $40.175 \pm 6.403$ & $0.272 \pm 0.155(-0.071)^{\mathrm{a}}$ \\
$\mathrm{DCM}$ & 10 & $38.667 \pm 4.939$ & $63.705 \pm 26.341(-0.604)$ \\
$\mathrm{D}+\mathrm{V}$ & 10 & $35.661 \pm 5.743$ & $23.876 \pm 18.385(0.133)$ \\
DCM+V & 10 & $37.665 \pm 6.256$ & $14.077 \pm 11.140(0.367)$ \\
\hline
\end{tabular}

a $>>0.05$. Results are expressed as the mean $\pm \mathrm{SD}$. 3-NT, 3-nitrotyrosine; AI, apoptotic index; r, correlation coefficient; $\mathrm{N}$, normal group; $\mathrm{DCM}$, diabetic cardiomyopathy group; $\mathrm{D}+\mathrm{V}$, diabetes + valsartan pretreatment group; $\mathrm{DCM}+\mathrm{V}$, diabetic cardiomyopathy + valsartan treatment group.

valsartan treatment, the rat hearts remained enlarged and the ratios of heart/body weight increased through the development of the DCM rat model. As the development period for DCM was short (12 weeks), neither myocardial ischemia due to severe narrowing of the coronary arteries nor heart congestion due to ventricle overload were observed in the DCM rats. Under the microscope, cardiomyocytes were assembled neatly, nucleus size was similar, plasma staining was even and plasma membranes appeared to be intact for the hearts of rats without DCM. However, in the hearts of rats with DCM, degeneration and necrosis were observed to some extent in the myocardial cells, with the proliferation of fibroblast cells between the inter-cardiomyocytes, an increase in collagen fibers and disordered cell assembly. The above pathological alterations led to dilated congested hearts and an increase in the heart ratios (heart/body weight), which is a comprehensive early response of cardiomyocytes to diabetes, leading to the development of heart failure in the late stages of DCM (8). Hyperglycemia was thought to trigger the above pathological process, but how to initiate and develop the degeneration and necrosis of cardiomyocytes and intercellular fibration was unknown; the data from the present study may provide the answer. Results of this study indicate that the AI of cardiomyocytes in the DCM, $\mathrm{D}+\mathrm{V}$ and $\mathrm{DCM}+\mathrm{V}$ groups were significantly higher than in the $\mathrm{N}$ group, and revealed associations between $\mathrm{AI}$ and the degeneration and necrosis of cardiomyocytes and intercellular fibration.
In 1972, Rubler et al (9) observed a special cardiomyopathy in diabetic patients without significant coronary atherosclerosis. Later, Hamby et al (10) proposed a new concept of DCM through further pathological diagnosis, and were the first to link DCM to the metabolism of diabetics. Up until 2000, studies reported necrosis in a great number of myocardial cells in diabetic patients, and the necrosis of the myocardial cells was more severe and significant if the diabetic patient also suffered from hypertension.

Previously, Ceriello (11) observed that under hyperglycemic conditions, overabundant ROS were provoked into bursting out from the endothelial cells, and were accompanied by higher levels of 3-NT and increases in collagen and fibronectin, which activated PKC- $\beta, \mathrm{NAD}(\mathrm{P}) \mathrm{H}$ oxidase and Bax. Hyperglycemia in the diabetic rats was well controlled in a normal range, but if it relapsed for two months, the levels of ROS and nitrogen and nitric oxide were inhibited by half and the levels of nitrotyrosine were $80 \%$ higher than in the control group. Our data agree with the above observations. In this study, the overexpression of 3-NT in myocardial tissue (EI) was a unique characteristic, and was observed to be significantly higher in the $\mathrm{DCM}, \mathrm{D}+\mathrm{V}$ and $\mathrm{DCM}+\mathrm{V}$ groups than in the $\mathrm{N}$ group. Furthermore, the profile of 3-NT EI was correlated positively to the profile of AI, where an increase in 3-NT was likely to provoke apoptosis in cardiomyocytes. Oxidative stress paved the way for apoptosis, cardiomyocyte necrosis and fibration. Hyperglycemia may exacerbate the damage caused by oxidative stress induced by angiotensin II. The oxidative product-3-NT in diabetic rats with DCM triggers a dysfunctional pathway of the mitochondrial and membrane receptors, which participate in the activation of the caspase-cascade pathway and Bcl-2 expression in apoptosis (12). 3-NT was a post-translational protein modification, and increased when accompanied by ROS and RNS. Turko and Murad (13) proposed that the production pathways of 3-NT may be both $\mathrm{ONOO}^{-}$- and non-ONOO--dependent. 3-NT production from these pathways caused the tyrosine residues of proteins or free tyrosine residues to nitrate, and feedback to enhance 3-NT production. The nitration of proteins may deactivate the protein enzymes and induce cellular necrosis and apoptosis.

There is increasing evidence $(14,15)$ that AT1 and AT2 produce excess oxygen or nitrogen radicals by reducing the nicotinamide adenine dinucleotide phosphate oxidase pathway, thereby resulting in oxidative stress, myocardial cell death and inflammation. In 2006, Cai et al (16) demonstrated, using a transgenic diabetic mouse model, that in the early stages of diabetes, high blood glucose levels are able to cause the heart to produce overabundant free oxygen or nitrogen radicals. ROS and RNS exhaust the capacity of myocardial inherent antioxidants, tipping the balance from antioxidant to oxidant, thereby leading to mitochondrial dysfunction and myocardial cell death via oxidative damage. When the number of myocardial cell deaths reaches this threshold, heart function cannot be replenished or compensated, resulting in heart failure.

In this study, after intervention with valsartan, an antagonist of the angiotensin II receptor, the EIs of 3-NT in the cardiomyocyte tissue of the $\mathrm{D}+\mathrm{V}$ and $\mathrm{DCM}+\mathrm{V}$ groups decreased compared with the DCM group. The number of cardiomyocyte apoptotic cells decreased, indicating that the expression of 3-NT in cardiac tissue was inhibited, whereas 
cardiomyocyte apoptosis was induced by 3-NT. It was demonstrated that 3-NT played a key role in oxidative damage in the development of DCM. The angiotensin II receptor pathway was inhibited by valsartan, whereby the inhibition of oxidative stress induced by hyperglycemia interrupted the production of 3-NT, and diminished cardiomyocyte apoptosis. Although the EI of 3-NT and apoptosis in cardiomyocytes were inhibited by valsartan, rat heart ventricles remained expanded and dilated, and the heart/weight ratios were increased. This demonstrated that cardiomyocyte necrosis was not the only mechanism producing dilated rat heart ventricles, as the inhibition of 3-NT expression diminished cardiomyocyte apoptosis but did not halt the ventricular dilation. In the diabetic state, the abnormal proliferation of myocardial extracellular matrix composed of collagen, non-collagenous glycoproteins and proteoglycans also contributed to the myocardial contractility, diastolic dysfunction and heart dilation (17). Further investigation into the effect of 3-NT on myocardial extracellular fibrosis and myocardial interstitial fibrosis is required to reveal the mechanism for DCM.

Ceriello et al (18) demonstrated that the nitro-tyrosine concentration in the plasma correlated positively with the blood glucose concentration in patients with type II diabetes. Angiotensin II receptor antagonists, such as irbesartan, may decrease 3-NT levels in diabetic patients, and may effectively counteract the formation of 3-NT in a state of acute hyperglycemia (19). However, in this study, no significant difference in 3-NT levels was found among the four different groups, and no correlation was found between the 3-NT levels in blood and the AI in myocardial tissue in the DCM group. After treatment with valsartan, there was no correlation between the 3-NT in the serum and the AIs in the DCM group.

This study demonstrated that overexpression of 3-NT in the myocardial tissues of rats in the DCM group was not associated with cardiomyocyte apoptosis; however, valsartan alleviated myocardial apoptosis in rats of the DCM group by inhibiting the expression of 3-NT in myocardial tissue. Blood 3-NT in the peripheral circulation did not reflect the actual content of 3-NT in myocardial tissue. The EI of 3-NT in myocardial tissue (but not blood 3-NT) was able to predict cardiomyocyte apoptosis. Cardiomyocyte apoptosis induced by 3-NT was alleviated by treatment with valsartan, but had no effect on the ventricular dilation in the hearts of rats with DCM, implying that cardiomyocyte apoptosis was not the only pathway leading to ventricular dilation in DCM.

\section{Acknowledgements}

The authors would like to express their gratitude to all the authors who reviewed this manuscript. This study was not supported by any grant sponsors.

\section{References}

1. Bell DS: Diabetic cardiomyopathy. Diabetes Care 26: 2949-2951, 2003.

2. Dokken BB: The Pathophysiology of Cardiovascular Disease and Diabetes: Beyond Blood Pressure and Lipids. Diabetes Spectr 21: 160-165, 2008.

3. Cai L and Kang YJ: Oxidative stress and diabetic cardimyopathy: a brief review. Cardiovasc Toxicol 1: 181-193, 2001.

4. Aksakal E, Akaras N, Kurt M, et al: The role of oxidative stress in diabetic cardiomyopathy: an experimental study. Eur Rev Med Pharmacol Sci 15: 1241-1246, 2011.

5. Cai L and Kang YJ: Cell death and diabetic cardimyopathy. Cardiovasc Toxicol 3: 219-228, 2003.

6. Srinivasan K, Viswanad B, Asrat L, et al: Combination of high-fat diet-fed and low-dose streptozotocin-treated rat: a model for type 2 diabetes and pharmacological screening. Pharmacol Res 52: 313-320, 2005.

7. Raviv G, Kiss R, Vanegas JP, et al: Objective measurement of the different collagen types in the corpus cavernosum of potent and impotent men: an immunohistochemical staining with computerized-image analysis. World J Urol 15: 50-55, 1997.

8. Tillquist MN and Maddox TM: Update on diabetic cardiomyopathy: inches forward, miles to go. Curr Diab Rep 12: 305-313, 2012.

9. Rubler S, Dlugash J, Yuceoglu YZ, et al: New type of cardiomyopathy associated with diabetic glomerulosclerosis. Am J Cardiol 30: 595-602, 1972.

10. Hamby RI, Zoneraich S and Sherman L: Diabetic cardiomyopathy. JAMA 229: 1749-1754, 1974.

11. Ceriello A: The hyperglycemia-induced metabolic memory: the new challenge for the prevention of CVD in diabetes. Rev Esp Cardiol 8 (Suppl C): 11-17, 2008

12. Kowluru RA: Effect of reinstitution of good glycemic control on retinal oxidative stress and nitrative stress in diabetic rats. Diabetes 52: 818-823, 2003.

13. Turko IV and Murad F: Protein nitration in cardiovascular diseases. Pharmacol Rev 54: 619-634, 2002.

14. Singh VP, Le B, Khode R, et al: Intracellular angiotensin II production in diabetic rats is correlated with cardiomyocyte apoptosis, oxidative stress, and cardiac fibrosis. Diabetes 57: 3297-3306, 2008.

15. Zhou G, Li X, Hein DW, et al: Metallothionein suppresses angiotensin II-induced nicotinamide adenine dinucleotide phosphate oxidase activation, nitrosative stress, apoptosis, and pathological remodeling in the diabetic heart. J Am Coll Cardiol 52: 655-666, 2008.

16. Cai L, Wang Y, Zhou G, et al: Attenuation by metallothionein of early cardiac cell death via suppression of mitochondrial oxidative stress results in a prevention of diabetic cardiomyopathy. J Am Coll Cardiol 48: 1688-1697, 2006.

17. Mason RM and Wahab NA: Extracellular matrix metabolism in diabetic nephropathy. J Am Soc Nephrol 14: 1358-1373, 2003.

18. Ceriello A, Mercuri F, Quagliaro L, et al: Detection of nitrotyrosine in the diabetic plasma: evidence of oxidative stress. Diabetologia 44: 834-838, 2001.

19. Ceriello A, Assaloni R, Da Ros R, et al: Effect of irbesartan on nitrotyrosine generation in non-hypertensive diabetic patients. Diabetologia 47: 1535-1540, 2004. 